\title{
PECULIARITIES OF DRUG-RESISTANT TUBERCULOSIS IN UKRAINE AND KHARKIV REGION
}

\author{
Shevchenko O., Matveyeva S., Stepanenko A., Choporova O., Pohorielova O. \\ Kharkiv National Medical University, Ukraine \\ https://doi.org/10.35339/ic.6.2.93-100
}

\begin{abstract}
Background. Multidrug-resistant tuberculosis and extensively drug-resistant tuberculosis remain severe epidemic problems in the world.

Therefore the purpose of our study was to investigate the dynamics of the incidence of multidrug-resistant and extensively drug-resistant tuberculosis, the structure of cases and the effectiveness of treatment in this category of patients in Kharkiv region and Ukraine. Subjects and methods. To perform the study, data from reporting forms No. 4-2 (TB $07-$ MDR TB), No. 8-6 (TB 08) and data from Kharkiv region and from other regions of Ukraine were processed. The form "TB 07 - MDR TB" shows the total number of MDR-TB and XDR-TB cases; new cases, relapses and other cases of re-treatment among them; number of MDR-TB and XDR-TB cases in which anti-tuberculosis treatment started. The form "TB 08" shows treatment results in MDR-TB and XDR-TB cases. We investigated MDR-TB and XDR-TB cases detected in 2015-2017. Statistical processing of information was carried out using Microsoft Office Excel 2010. Results. The stable dynamics of drug-resistant tuberculosis incidence at the level 12.3-12.5 per 100000 population was found in Kharkiv region while in Ukraine this parameter increased from 15.9 to 19.7 per 100000 population. In the structure of MDR-TB and XDR-TB cases, patients with firstly diagnosed tuberculosis prevailed: $46.7-54.4 \%$ in Kharkiv region and $45.0-51.2 \%$ in Ukraine. The patients with acquired drug resistance had relapse of tuberculosis or other cases of repeated treatment more often and in Kharkiv region their percentage prevailed significantly $(\mathrm{p}<0.01)$. Treatment effectiveness of firstly diagnosed drug-resistant tuberculosis $(58.2 \%$ in Kharkiv region and $55.8 \%$ in Ukraine) was significantly higher $(\mathrm{p}<0.01)$ than treatment effectiveness of cases of repeated treatment (45.4\% in Kharkiv region and 38.6\% in Ukraine).Conclusions. Early introduction of new effective molecular-genetic and cultural methods of drug-resistance diagnosis in Kharkiv region allowed to detect most of drug-resistant TB cases while in other regions of Ukraine these methods were introduced later that caused increase in MDR-TB and XDR-TB incidence during observation period. Domination of firstly diagnosed MDR$\mathrm{TB}$ and XDR-TB cases indicates critical epidemiological situation, as there are many undetected cases of drug-resistant tuberculosis that can infect people and increase incidence of primary resistant tuberculosis. Higher treatment effectiveness in Kharkiv region can be explained by accessibility of new antituberculous second-line drugs. Thus, wide introduction of methods of early drug-resistance detection and accessibility of new antituberculous drugs improve epidemiological situation on tuberculosis in Kharkiv region compared with other regions of Ukraine.
\end{abstract}

Keywords: Tuberculosis, MDR-TB, XDR-TB, treatment effectiveness.

\section{Introduction}

In 2015, the WHO announced the "End TB" strategy for overcoming the epidemic of tuberculosis in the world. According to the

Corresponding Author:

Olha Pohorielova MD, PhD student of the

Department of Phthisiology and Pulmonology.

Kharkiv National Medical University, Ukraine.

E-mail: evildevilolga@gmail.com objectives of this strategy, it is necessary to reduce the mortality rate by $95 \%$ and the incidence rate by $90 \%$ by 2035 . To do this, it is necessary to achieve the effectiveness of treatment of tuberculosis with a preserved sensitivity of $85 \%$ and multidrug-resistant tuberculosis - 75\% [1].

However, drug-resistant tuberculosis, and in particular multidrug-resistant (MDR-TB) and extensively drug-resistant tuberculosis (XDR-TB), 
is now a complex epidemiological problem in the world. According to the WHO, in 2017, there were 160,684 new cases of MDR-TB and XDR-TB, which is $4.9 \%$ more than in 2016 . Of these, only $87 \%$ of patients began treatment with secondline anti-TB drugs. At the same time, the effectiveness of the treatment of drug-resistant tuberculosis is only $55 \%$ [2].

In the Eastern European region, 9 countries with the highest burden of MDR-TB include Azerbaijan, Belarus, Kazakhstan, Kyrgyzstan, Moldova, Russian Federation, Tajikistan, Ukraine and Uzbekistan. According to the WHO reports, all patients in the region had access to secondline drugs [3]. At the same time, one of the five cases of MDR-TB in the world is registered in European Region. The average effectiveness of treatment for MDR-TB in the region is 51\% [4].

\section{Purposes, subjects and methods:}

2.1. Purpose - to investigate the dynamics of the incidence of multidrug-resistant and extensively drug-resistant tuberculosis, the structure of cases and the effectiveness of treatment in this category of patients in Kharkiv region and in Ukraine.

\subsection{Subjects \& Methods}

The observation period 2015-2017 was chosen because treatment results can be evaluated only in 18-20 months according to MDR-TB and XDR-TB treatment regimens (2017-2019 respectively). Kharkiv region was chosen for the detailed analysis due to both high population density and migration that causes favorable conditions for the tuberculosis distribution.

On the other hand, there it is an appropriate access to the TB diagnosis and treatment that can control of tuberculosis in the region. Kharkiv region is among first ones that supported innovations in the framework of the National Tuberculosis Control Program. Therefore, each patient has access to molecular-genetic methods for diagnosing tuberculosis and M.tuberculosis resistance (GeneXpert MTB/RIF, Hain MDR TB), new cultural diagnostic methods (BACTEC MGIT 960), new anti-tuberculosis drugs for the treatment of drug-resistant tuberculosis. To perform the study, data from reporting forms No. 4-2 (TB 07 - MDR TB), No. 8-6 (TB 08) from Kharkiv region and from other regions of Ukraine were processed. The form "TB 07 - MDR TB" shows the total number of MDR-TB and XDRTB cases; new cases, relapses and other cases of re-treatment among them; number of MDRTB and XDR-TB cases in which anti-tuberculosis treatment started. The form "TB 08" shows treatment results in MDR-TB and XDR-TB cases. The proportion of resistant forms among various types of tuberculosis cases was calculated. Types of tuberculosis cases were determined in accordance with the Order of the Ministry of Health of Ukraine No. 620 as follows: firstly diagnosed tuberculosis (FDTB) - patients who had not previously been diagnosed with tuberculosis and who had not received antituberculosis treatment previously, relapse of tuberculosis (RTB) - patients who successfully completed earlier treatment for tuberculosis, other cases of re-treatment - treatment after interruption, treatment after treatment failure, and other cases that are not included in the classification. The treatment was considered as effective when the bacterial excretion stopped and the severity of clinical and radiological manifestations of the disease was decreased. Resistance type was determined as multidrugresistant tuberculosis (MDR-TB) - resistance to isoniazid and rifampicin (main first-line drugs) and extensively drug-resistant tuberculosis (XDRTB) - resistance to isoniazid, rifampicin, one of the fluoroquinolones and one of the aminoglycosides. Statistical processing of the information was carried out by using Microsoft Office Excel 2010.

\section{Ethics approval and consent to participate}

An epidemiological study was performed using standard reporting forms, without the direct participation of patients. Personal data were not used and the consents were not signed.

Conflict of interests. The authors declare that they have no competing interests.

\section{Results and discussion}

The dynamics of the incidence of MDR-TB and XDR-TB in Kharkiv region was analyzed; a stable level of $12.3-12.5$ per 100000 population in 2015 and 2017 and a sharp increase in the incidence of up to 13.8 per 100000 population in 2016 were revealed. In Ukraine, this level was 15.9 per 100000 population in 2015 and steadily grew by $15.1 \%$ in 2016 (18.3 per 100000 population) and by $23.9 \%$ in 2017 (19.7 per 100000 population).

Since the sources of multidrug resistance and extensively-drug resistance may be primary infection with resistant strains (primary resistance), as well as the acquisition of secondary resistance due to the recurrence of tuberculosis with a second course of tuberculosis chemotherapy, treatment failure or treatment interruption, the proportion of different cases 
among patients with drug-resistant tuberculosis was investigated.

According to the type of case (first diagnosed tuberculosis or relapse of tuberculosis) in Kharkiv region, patients were distributed as follows: in 2015 , the proportion of patients with FDTB was $46.7 \%$, patients with RTB $-29.1 \%$, patients with other cases of repeated treatment $-21.2 \%$. In 2016, there was a significant increase in the proportion of patients with FDTB $-54.4 \%$, RTB $32.2 \%(\mathrm{p}=0.04)$ and a decrease in the proportion of patients with other cases of repeated treatment $13.4 \%(p=0.01)$. In 2017, the proportion of patients with FDTB was $57.4 \%$, RTB - 28.9\%, other cases of re-treatment $-13.7 \%$.

In Ukraine, there was a similar trend. In 2015, FDTB accounted for $45.0 \%$ of cases, RTB $29.6 \%$, and other cases of re-treatment $-25.4 \%$. In 2016, patients with FDTB accounted for $50.1 \%$, with RTB $-31.1 \%$, with other cases of repeated treatment $-18.8 \%$. In 2017 , FDTB $51.2 \%$, RTB $-30.9 \%$, other cases of re-treatment 17.9\% (Fig. 1, 2).

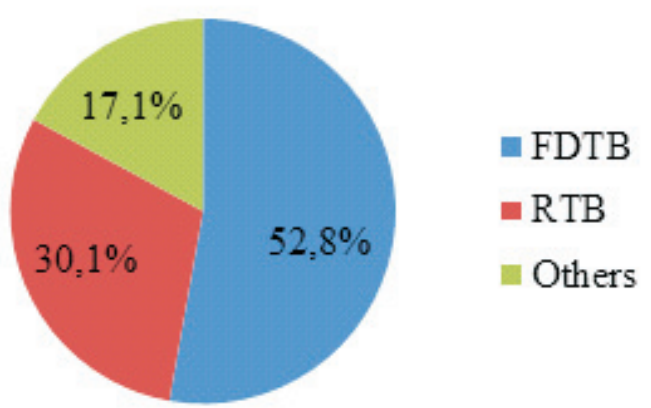

Fig. 1. Distribution of various cases of tuberculosis among patients with drug-resistant in Kharkiv region

FDTB - firstly diagnosed tuberculosis RTB - relapse of tuberculosis

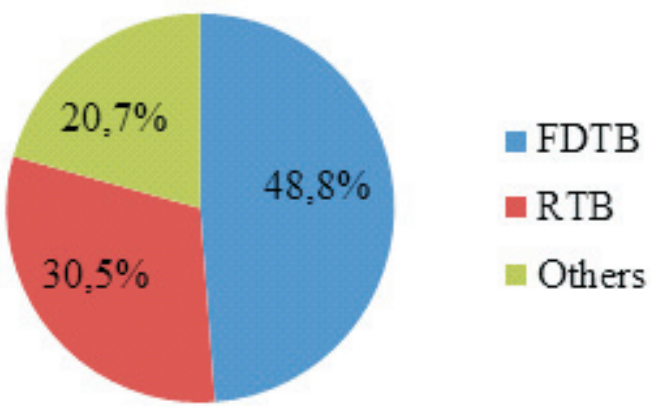

Fig. 2. Distribution of various cases of tuberculosis among patients with drug-resistant in Ukraine

FDTB - firstly diagnosed tuberculosis RTB - relapse of tuberculosis
The difference between Kharkiv region and Ukraine in distribution of various cases of tuberculosis among patients with drug-resistance was found only in part of other cases of re-treatment in $2016(p=0.01)$ and FDTB cases $(p=0.03)$ in 2017 . The difference was insignificant $(p>0.05)$ in other cases.

We analyzed the proportion of patients with the primary susceptible tuberculosis (first diagnosed or relapsed) with acquired drug resistance, who transferred to the treatment by the second-line drugs. In Kharkiv region, there was insignificant increase in the percentage of patients with FDTB in whom M. tuberculosis (MTB) developed acquired drug-resistance (2015 $-17.3 \%, 2016-21.6 \%, 2017-22.6 \%(p=0.09))$ and with other cases of repeated treatment $(2015$ - 30, 8\%, $2016-32.3 \%, 2017-33.1 \%)$. The worst situation with a slightly positive trend was observed in patients with RTB (2015 - 59.4\%, $2016-57.1 \%, 2017-51.1 \%(\mathrm{p}=0.03))$. Thus, we can see that acquired drug resistance is usually developed in patients with relapses of tuberculosis and other cases of re-treatment $(\mathrm{p}<0.01)$.

In Ukraine, there was a similar situation. Among patients with FDTB, $14.2 \%$ of patients were transferred to treatment with second-line drugs in 2015, in 2016 - 18.3\%, in 2017 - 18.0\%. The proportion of patients with other cases of re-treatment transferred to treatment with second-line drugs was: in 2015 - 23.5\%, in 2016$18.3 \%$, in $2017-27.1 \%$. Patients with RTB were transferred to treatment with second-line drugs in $2015-43.3 \%$ of cases, in $2016-41.9 \%$, in 2017 - 43.7\% (Fig. 3).

The part of TB relapses and other cases of the re-treatment among the cases of acquired drug-resistance are significantly prevalent in Kharkiv region $(\mathrm{p}<0.01)$.

We also analyzed the distribution of both types of resistance (MDR-TB and XDR-TB) and their dynamics in Kharkiv region.

Among the patients with FDTB, in 2015 MDR-TB accounted for 91.3\%, XDR-TB $8.7 \%$, in $2016-90.7 \%$ and $9.3 \%$, in $2017-90.7 \%$ and $9.3 \%$ respectively.

Among the patients with RTB, in 2015 MDRTB accounted for $87.2 \%$, XDR-TB - for $12.8 \%$, in $2016-90.7 \%$ and $9.3 \%$, in $2017-77.7 \%$ and $22.3 \%$, respectively.

Among the patients with other cases of retreatment, in 2015 MDR-TB accounted for $89.4 \%$, XDR-TB - for $10.6 \%$, in $2016-79.1 \%$ and $20.9 \%$, in $2017-75.6 \%$ and $24.4 \%$ respectively. (Fig. 4). 


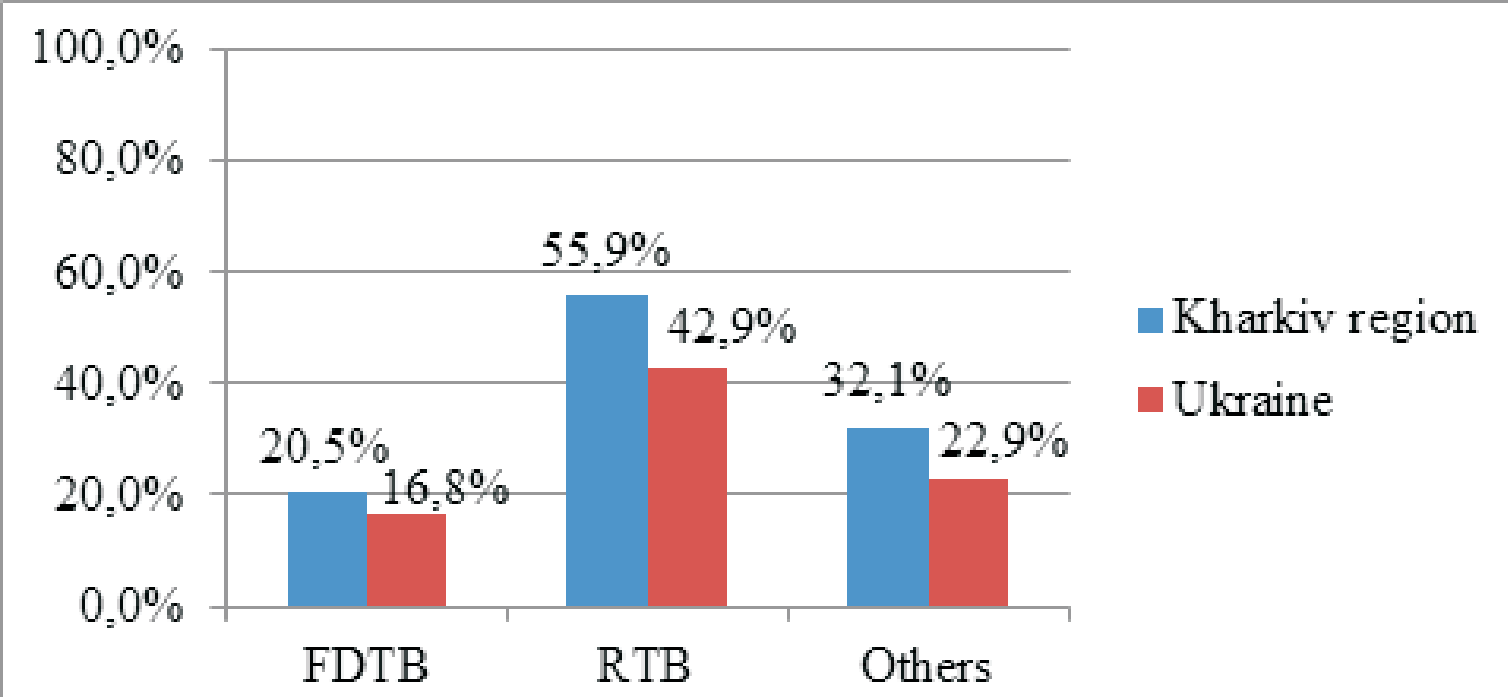

Fig. 3. The proportion of patients with various cases of tuberculosis that were transferred to treatment with second-line drugs: FDTB - firstly diagnosed tuberculosis, $\mathrm{RTB}$ - relapse of tuberculosis

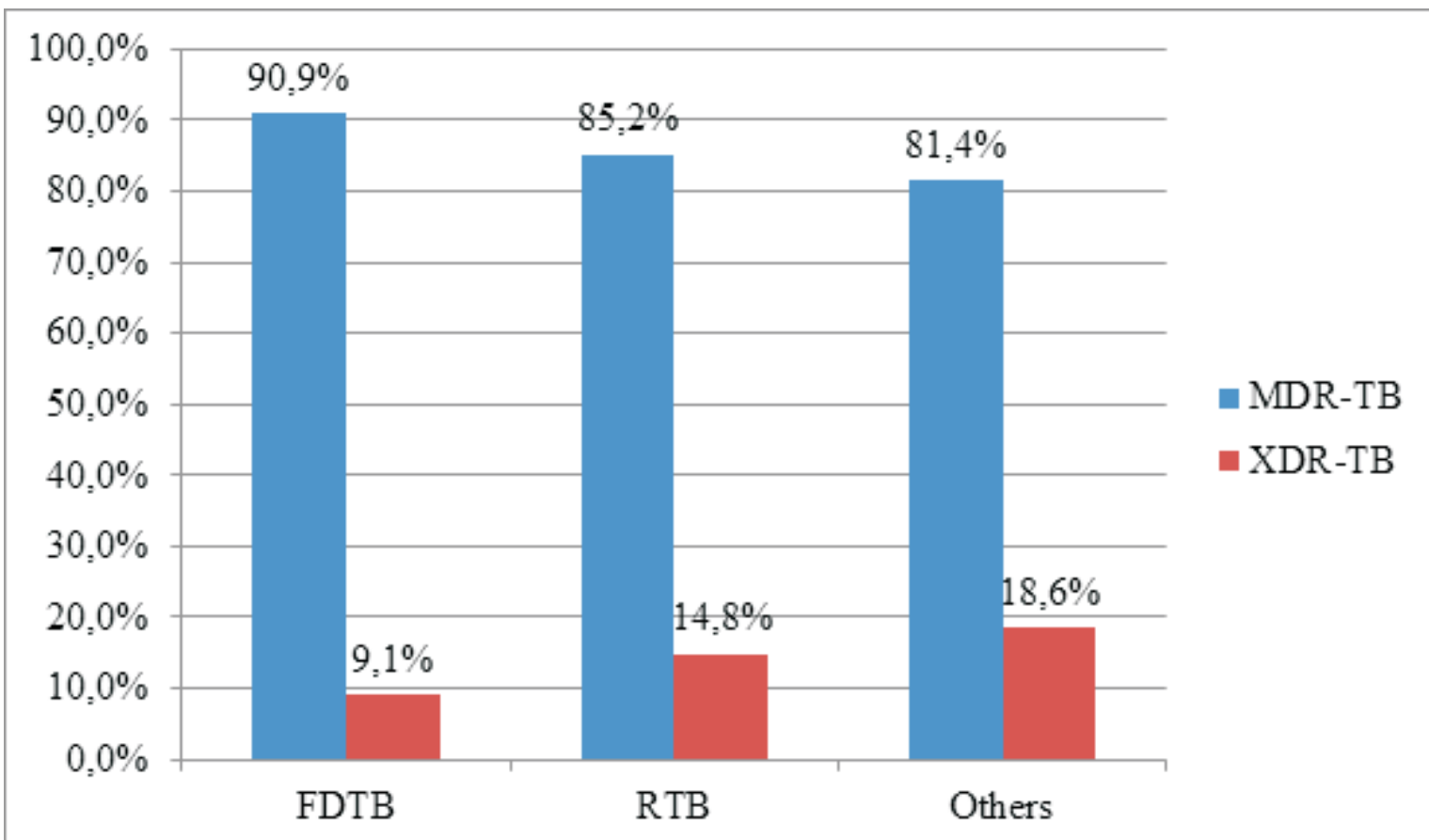

Fig. 4. The structure of $M$. tuberculosis resistance in patients with various cases of tuberculosis: FDTB - firstly diagnosed tuberculosis, RTB - relapse of tuberculosis, MDR-TB - multidrug-resistant tuberculosis, XDR-TB - extensively-drug resistant tuberculosis

Thus, we can see that XDR-TB is observed in cases of relapses and re-treatment more often than in FDTB cases $(\mathrm{p}<0.01)$.

Since MTB resistance profile is directly related to the effectiveness of treatment, the effectiveness of treating various cases of tuberculosis in patients transferred for treatment with second-line drugs in Kharkiv region was compared.

The effectiveness of treatment of MDR-TB among FDTB was: in 2015 - 56.9\%, $2016-$
$65.8 \%, 2017-51.8 \%$ with a corresponding mortality rate of $21.8 \%, 12.1 \%$ and $14.5 \%$. The effectiveness of treatment of MDR-TB among RTB was: in 2015-54.3\%, 2016-60.0\%, $2017-$ $60.4 \%$ with a corresponding mortality rate of $26.8 \%, 13.8 \%$ and $19.8 \%$. The effectiveness of treatment of MDR-TB among other cases of repeated treatment was: in $2015-33.5 \%, 2016-$ $33.3 \%, 2017-30.8 \%$ with a corresponding mortality rate of $31.6 \%, 28.0 \%$ and $15.4 \%$ (Fig . 5). 


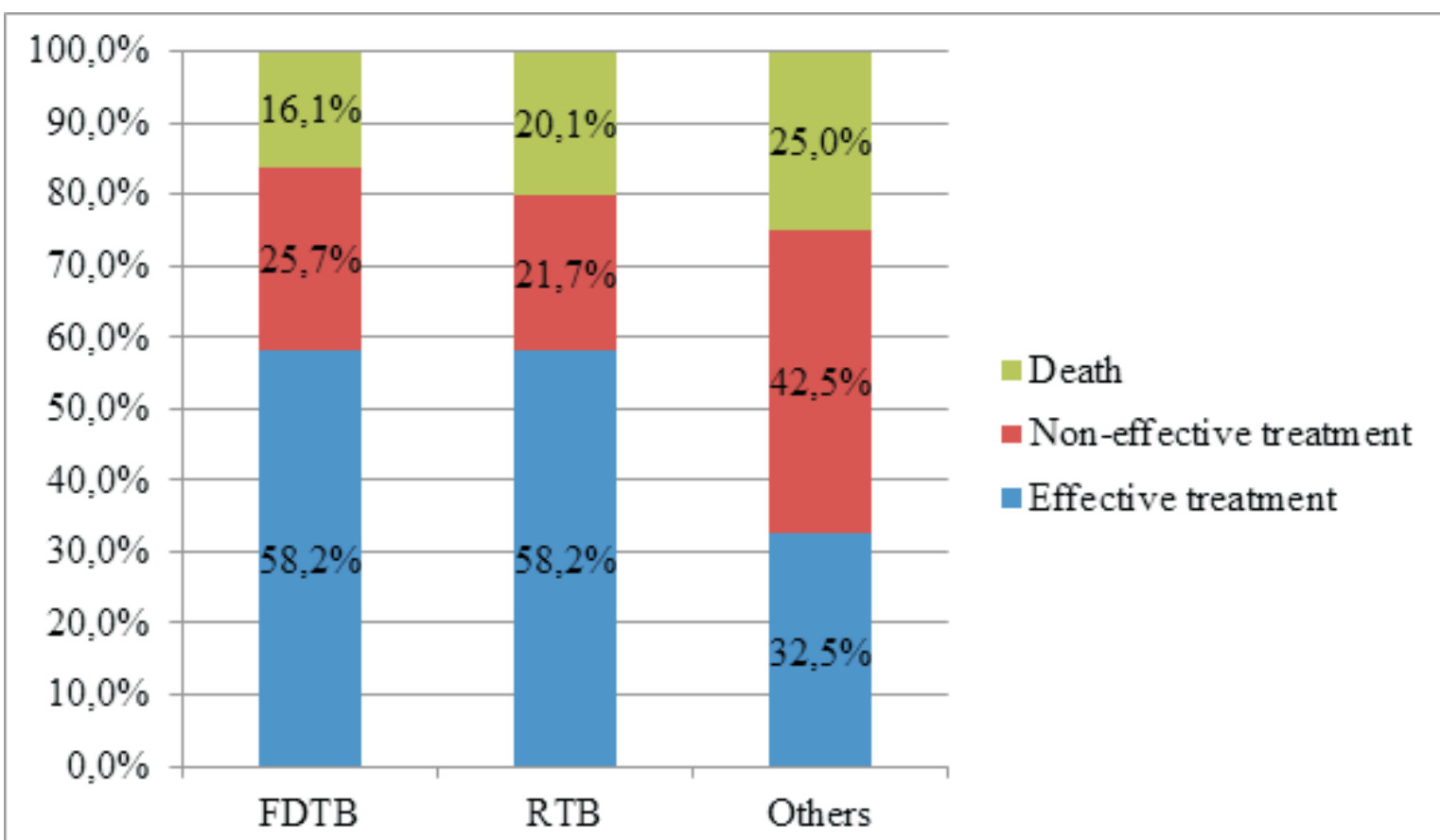

Fig. 5. Efficacy of MDR-TB treatment in patients with various types of tuberculosis cases: FDTB - firstly diagnosed tuberculosis, RTB - relapse of tuberculosis

There was no difference between MDR-TB relapse and FDTB cases treatment effectiveness $(p>0.05)$ but the treatment effectiveness of other cases of repeated treatment was significantly lower $(\mathrm{p}<0.01)$ which led to higher case fatality rate in this group $(\mathrm{p}=0.01)$.

We compared the treatment effectiveness in patients with different types of MDR-TB and XDR-TB in Ukraine. The effectiveness of treatment of the firstly diagnosed MDR-TB cases significantly increased from $50.6 \%$ in cases detected in 2015 to $55.8 \%$ in 2016 and to $61 \%$ in 2017 ( $p<0.01)$ with a corresponding case fatality rate of $16.8 \%, 15.3 \%$ and $14.2 \%$ which also significantly decreased $(\mathrm{p}<0.01)$. The effectiveness of treatment of relapsed cases of MDR-TB in 2015 was: $33.2 \%$, in $2016-39.9 \%$, in 2017 $42.7 \%(\mathrm{p}<0.01)$ with a corresponding case fatality of $24.0 \%, 17.7 \%$ and $16.8 \%(\mathrm{p}<0.01)$. Treatment effectiveness of FDTB MDR-TB cases was significantly higher $(p<0.01)$ than in cases of repeated treatment with corresponding lower case fatality rate $(\mathrm{p}<0.01)$.

XDR-TB treatment effectiveness in patients with FDTB is significantly increased from $37.9 \%$ cases detected in 2015 to $43.1 \%$ - in 2016 and to $51.2 \%$ - in $2017(\mathrm{p}<0.01)$ with the corresponding fatality rate $-16.7 \%, 18.2 \%$ and $20.0 \%$, relevantely. XDR-TB treatment effectiveness in patients with the relapses did not changed significantly and was $28.2 \%$ in $2015,21.5 \%$ in 2016 and $32.4 \%$ in 2017 $(\mathrm{p}=0.12)$ with a corresponding case fatality rate -
$21.4 \%, 27.7 \%$ and 0 , relevantely (Fig. 6, 7).

Treatment effectiveness of the newly diagnosed MDR-TB cases was significantly higher $(\mathrm{p}<0.01)$ but the case fatality rate in new cases and cases of re-treatment was almost same $(\mathrm{p}=0.14)$.

Discussion. MDR-TB and XDR-TB remain a serious problem not only in Ukraine, but all over the world [5-9]. However, the increase in the incidence of drug-resistant tuberculosis in Ukraine can be explained not only by a poor epidemiological situation, but also by improved diagnosis, since now molecular-genetic research methods such as GeneXpertMTB/RIF and rapid culture methods of research (BACTEC) have become available even to the residents of remote villages. In Kharkiv region, the widespread introduction of rapid methods for diagnosing drugresistance became available as early as 20132014, thus a stable incidence rate of drug-resistant tuberculosis among residents of Kharkiv region was received.

But the direct evidence of the unfavorable epidemiological situation of drug-resistant tuberculosis in the country was the determination of the structure of tuberculosis cases. It turned out that among patients with MDR-TB and XDR$\mathrm{TB}$, primary drug resistance prevailed and increased, that is, MTB resistance to antituberculosis drugs in patients who had never received anti-tuberculosis treatment before. It means that epidemiological situation on drug- 


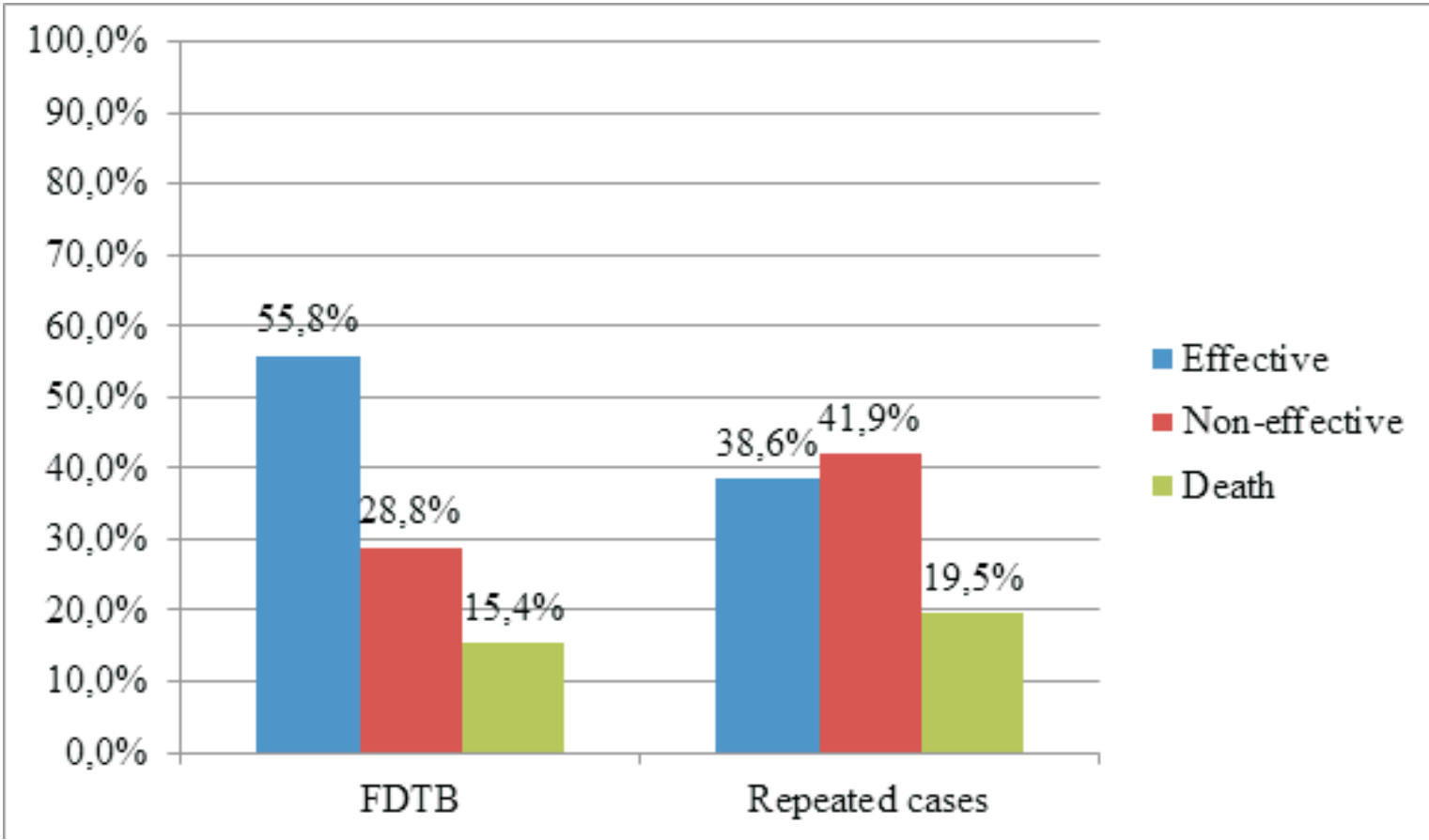

Fig. 6. Treatment effectiveness of MDR-TB: FDTB - firstly diagnosed tuberculosis

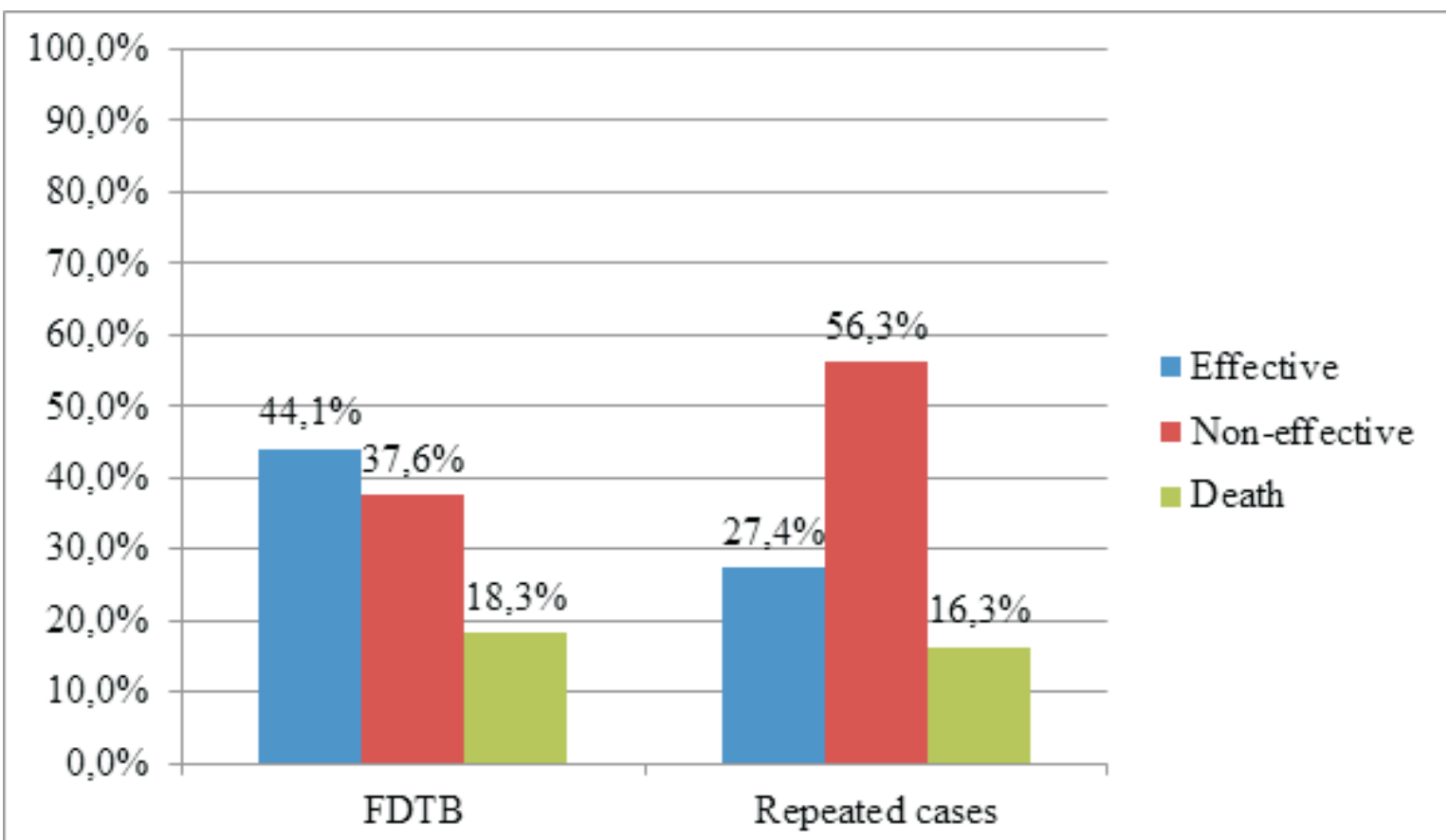

Fig. 7. Treatment effectiveness of XDR-TB: FDTB - firstly diagnosed tuberculosis

resistant tuberculosis in both Kharkiv region and Ukraine is critical so that non-detected patients with drug-resistant tuberculosis infect healthy people in household and casual contacts and increase part of firstly diagnosed MDR-TB and XDR-TB. Similar problem was also observed in number of other countries, that worsens the prognosis of treating patients and indicating an unfavorable epidemiological situation [10-12].
One of the key moments in preventing development of drug-resistance is administration of the effective chemotherapy regimen to the patients with tuberculosis. We have noticed that patients who started treatment from susceptible tuberculosis develop drug resistance more often after the relapse of tuberculosis or another case of the re-treatment (after treatment failure of treatment interruption). The repeated treatment 
course is resulting treatment failure in both Kharkiv region and Ukraine. It was assumed that it happens due to the fact that the MTB has enough time to acquire drug resistance during the second course of treatment. The situation is aggravated by the fact that for the treatment of FDTB and RTB, the same regimens and the same treatment duration are used, which increases the chances of the MTB for the development of drug-resistance [13-17]. Now we can propose treatment regimens with new antituberculous drugs (Bedaquiline and Delamanid) for these patients in Kharkiv region but results of new treatment regimens can not be evaluated yet.

Interesting data were obtained by analyzing separately the proportion of patients with MDRTB and XDR-TB. Although a greater amount of acquired drug-resistance falls on the recurrence of tuberculosis, most of the XDR-TB account for other cases of re-treatment, including treatment failure and interrupted treatment. This means that the main link in the prevention of the expansion of M.tuberculosis resistance is the administration of the adequate chemotherapy regimens and strict adherence by patients to the rules for administering anti-tuberculosis drugs. Effectiveness of administration of appropriate treatment regimens as well as introduction of more effective antituberculous drugs (new fluoroquinolones, Linezolid) is confirmed by the growth of newlydiagnosed XDR-TB cases treatment effectiveness.

\section{Conclusions}

The drug-resistant tuberculosis incidence was stable in Kharkiv region and increased in Ukraine during the observation period due to the fact of the new effective methods of drug resistance diagnosis (BACTEC MGIT 960, GeneXpert MTB/RIF, Hain MDR TB), that were introduced in Kharkiv region earlier and allowed to detect most of MDR-TB and XDR-TB cases.

Newly diagnosed MDR-TB and XDR-TB cases are prevailed in both Kharkiv region and Ukraine that means we still have many nondetected cases that can infect people and increase the incidence of primary resistant tuberculosis.

Acquired drug-resistance among patients with newly diagnosed tuberculosis was observed in Kharkiv region less often than in Ukraine. It can be explained by the earlier introduction of new anti-tuberculosis drugs in Kharkiv region as well as by accessibility of methods of early drugresistance detection. These factors lead to the increase of treatment effectiveness.

It was proven that repeated courses of treatment after the treatment failure due to treatment interruption regardless of the resistance profile result in treatment failure or death more often. Therefore, introduction of new antituberculosis drugs that can decrease number of first-course treatment failures and introduction of short treatment regimens for MDR-TB cases that can decrease number of treatment interruptions is a priority of the Ukrainian National Tuberculosis Control Program.

List of abbreviations:

FDTB - firstly diagnosed tuberculosis

RTB - relapse of tuberculosis

MDR-TB - multidrug-resistant tuberculosis

XDR-TB - extensively drug-resistant tuberculosis TB - tuberculosis

\section{References}

1. World Health Organization Fact sheet: Post-2015 Global TB Strategy and targets (2015). Available from: http://www.who.int/tb/post2015_strategy/en/.

2. Global Tuberculosis Report. World Health Organization (2018), 277 p.

3. World Health Organization Fact sheet: Tuberculosis in the WHO European Region (2018). Available from: http://www.euro.who.int/ data/assets/pdf file/0020/365150/wtbd-2018-eng.pdf?ua=1.

4. Tuberculosis surveillance and monitoring in Europe. World Health Organization (2017), $162 \mathrm{p}$.

5. Fojo, A.T., Dowdy, D.W. (2017).Multidrug-resistant tuberculosis in India: looking back, thinking ahead. Lancet Pub Health, 2, e8-e9. doi:10.1016/S2468-2667(16)30040-8.

6. Marais, B.J., Sintchenko, V. (2016) Epidemic spread of multidrug-resistant tuberculosis in China. Lancet Infect Dis, 17, 238-239. doi:10.1016/S1473-3099(16)30526-6.

7. Yang, C., Luo, T., Shen, X., Wu, J., Gan, M., Xu, P., ... Gao, Q. (2017). Transmission of multidrug-resistant Mycobacterium tuberculosis in Shanghai, China: a retrospective observational study using whole-genome sequencing and epidemiological investigation. Lancet Infect Dis, 17, 275-284. doi:10.1016/S1473-3099(16)30418-2. 
8. Cohen, K.A., Mansin, A.L., Abeel, T., Desjardins, C., Chapman, S.B., Hoffner,S., ... Earl, A.M. (2019). Extensive global movement of multidrug-resistant M. tuberculosis strains revealed by wholegenome analysis. Thorax, 1, 1-8 doi: 10.1136/thoraxjnl-2018-211616

9. Bai, R., Chi, S., Li, X., Dai, X., Ji, Z., Jian, M., ... Bao, F. (2019). Genetic Diversity and Drug Susceptibility Patterns of the Mycobacterium tuberculosis Complex in Yunnan, China. Bioscience Reports, 39. doi: 10.1042/BSR20181746

10. Chattu, K., Kumary, V. (2015). The Growing Epidemic of MDR- TB and Concerns for Global Health Security. International Journal of Contemporary Medical Research, 3, 329-31.

11.Xu, J., Wang, B., Hu, M. et al. (2017). Primary clofazimine and bedaquiline resistance among isolates from patients with multidrug-resistant tuberculosis. Antimicrob Agents Chemother, 61, e0023917. doi: 10.1128/AAC.00239-17

12.Migliori G.B., Sotgiu G., Gandhi N.R., Falzon, D., DeRiemer, K., Centis, R., ... Menzies, D. (2013). Collaborative Group for Meta-Analysis of Individual Patient Data in MDR-TB. Drug resistance beyond XDR-TB: results from a large individual patient data meta-analysis. Eur Respir J, 42, 169-179. doi: $10.1183 / 09031936.00136312$

13.Li, D., Wang, J., Ji, B.-Y., Cui, J-Y., Pan, X.L., Fan, C.L., ... Linh, H. (2015). Persistently high prevalence of primary resistance and multidrug resistance of tuberculosis in Heilongjiang Province, China. BMC Infectious Diseases, 26, 516. doi: 10.1186/s12879-016-1848-9

14.Dobler, C.C., Korver, S., Batbayar, O., Nyamdulam, B., Oyuntsetseg, S., Tsolmon, B., ... Marais, B.J. (2015). Multidrug-Resistant Tuberculosis in Patients for Whom First-Line Treatment Failed, Mongolia, 2010-2011. Emerg Infect Dis, 21, 1451-1454. doi: 10.3201/eid2108.141860.

15.Kumar, A., Singh, A.K., Upadhyay, V., Pandey, J. (2018). Epidemiology of multi-drug-resistant tuberculosis in Northern India. Biomedical and Biotechnology Research Journal, 2, 112-121. doi: 10.4103/ bbrj.bbrj_26_18

16.Caminero, J.A., Cayla, J.A., Garcia-Garcia, J., Garcia-Perez, F.J., Palacios, J.J., Ruiz-Manzano, J. (2017) Diagnosis and Treatment of Drug-Resistant Tuberculosis. Bronconeumologia, 9, 471-534. doi: 10.1016/j.arbres.2017.02.006

17.Jan, F., Wali, S., Sadia, S., Akbar, M.T., Akbar, H.S., Ahmad, W., ... Khalid, A. (2018). Drug resistance pattern in Mycobacterium tuberculosis to the first line drugs of pulmonary tuberculosis patients at Hazara Region, Pakistan. Tuberculoz we Toraks, 66(1), 26-31. doi: 10.5578/tt.60781

Received: 20-Mar-2019

Accepted: 11-Jun-2019 